\title{
Environment Simulation for a Continuous Mining Machine
}

John Albert Horst

Intelligent Systems Division

U.S. DEPARTMENT OF COMMERCE Technology Administration National Institute of Standards and Technology

Bldg. 220 Rm. B124

Gaithersburg, MD 20899 



\section{Environment Simulation for a Continuous Mining Machine}

John Albert Horst

Intelligent Systems Division

U.S. DEPARTMENT OF COMMERCE Technology Administration

National Institute of Standards

and Technology

Bldg. 220 Rm. B124

Gaithersburg, MD 20899

June 1994

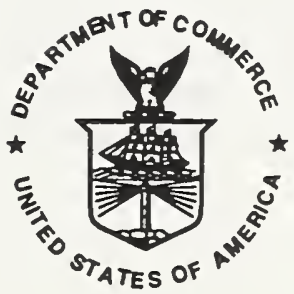

U.S. DEPARTMENT OF COMMERCE Ronald H. Brown, Secretary

TECHNOLOGY ADMINISTRATION

Mary L Good, Under Secretary for Technology

NATONAL INSTITUTE OF STANDARDS

AND TECHNOLOGY

Arat Prabhakar, Director 



\author{
John Albert Horst ${ }^{1}$
}

\begin{abstract}
A simulator is described for the motion of a continuous mining machine (CM) in a coal mine. Of special interest is the motion of the CM following collision with an immovable obstacle in the mine. This type of constrained motion effected our choice of representation of the CM and its environment. The CM is represented as a two dimensional polygonal hull and the mine obstacles are given two separate representations, certainty grids and obstacle boundary curves, each of which efficiently perform specific tasks. The object boundary curve representation is most useful for determining motion following a collision and the certainty grid is most useful for determining whether and where a collision has occurred.
\end{abstract}

ABSTRACT

\section{INTRODUCTION AND BACKGROUND}

The US Bureau of Mines (USBM) has developed a multiprocessing network architecture for low level servo control of the appendages of a continuous mining machine, called BOMNET, which is based on the BITBUS network communications standard [Schiffbauer 92]. Decision state graphs, based on the NIST's Real-time Control System (RCS) paradigm, have also been developed that can control the continuous mining machine (CM) through the BOMNET and allow operator interface as required. The RCS paradigm specifies hierarchical, heterogeneous levels of control where each level has a characteristic spatial and temporal resolution for the critical components of the system at that level [Horst 93]. These critical system components are control, sensing, and world modeling.

It has become apparent that simulation of the performance of the CM and the BOMNET (as it commands the CM to perform various tasks) would provide a useful and cost effective tool to researchers. This paper addresses the problem of $\mathrm{CM}$ motion simulation.

\footnotetext{
${ }^{1}$ Electronics engineer.

Robot Systems Division, The National Institute of Standards and Technology (NIST), U.S. Department of Commerce.
}

We are especially interested in the motion that occurs when the machine is constrained. This is common in a coal mine, where, for example, an operator will move a $\mathrm{CM}$ by intentionally colliding with 'ribs' 2 of coal in order to achieve a certain position and orientation. This research focusses on simulating constrained motion of the CM. We call this environment simulation, because we need to simulate the CM and aspects of its environment relevant to its motion. For example, the condition of the mine floor greatly effects CM motion.

A question arose early in the research, namely, should we do kinematics-based or kinetics-based simulation? If the effort is not overwhelming and the level of fidelity requires it, full description in terms of forces and torques (i.e., kinetics-based) is preferred. However, we discovered that the effort to model forces and torques accurately was a task beyond our requirements and resources. Kinematics-based simulation produced a sufficient level of fidelity. Therefore, we proceeded with the development of a kinematics-based simulator.

\footnotetext{
2 Walls of unmined coal are called ribs.
} 


\section{COMPONENTS OF CM POSITION/MINE ENVIRONMENT SIMULATOR}

This paper describes work done to create an environment simulator that will simulate the motion dynamics of the $\mathrm{CM}$ and the interaction with obstacles in an underground coal mine. However, it is also figure 1. This collision detection algorithm simply samples the simulated pose at a high rate and senses overlap of CM hull points with occupied regions of the simulated true map. The occupancy probabilities for any cell in the map are zero or one and there is no checking with neighboring cells for an 'impending' collision. When the entire control system is made operational, this simulator will be inactive.

In the operational world model (servo level in figure 1), collision is detected by a commanded value accompanied by an 'abnormal' response in the position and orientation trajectory of the CM. For example, if the $\mathrm{CM}$ is commanded to move forward, no detected motion would be abnormal. This collision detection algorithm could also act as a predictor, looking at the occupancy probability (above some threshold) of cells in the 'best-estimate-map' near the finite set of points on the CM hull, in world coordinates. We envision, as well, that collision detection

important to consider how it will interface with other portions of the existing and planned control and simulation system for computercontrolled coal cutting with a CM [Schiffbauer 92]. Such considerations are illustrated in figure 1. The components of the $\mathrm{CM}$ and mine environment simulator consist of the mine map, description and location of the CM, the motion controller (issuing commands to the CM through the BOMNET), and the pose sensor processing module. These will all be real modules eventually and the simulators need to be considered as separate entities. However, we don't anticipate eliminating the simulated modules even after commencing real testing, since they are useful for failure diagnostics during real operation.

This paper describes simulated collision detection only as shown at the bottom level of simulation might continue within the world model when the system is operational as a safety and system performance check.

\section{REPRESENTATION}

Before we choose methods of representation for the mine obstacles and the CM, we must consider how these representations are to be used as well as what the system and computational constraints are. We need to detect when the CM is nearing a collision and/or has collided with an obstacle. Additionally, we must be able to simulate the motion of the CM after it makes such a collision under a variety of motion commands. The representation choices are discussed in sections 3.1 and 3.2.

With minimal loss of fidelity, we can restrict the spatial representation of the CM to 


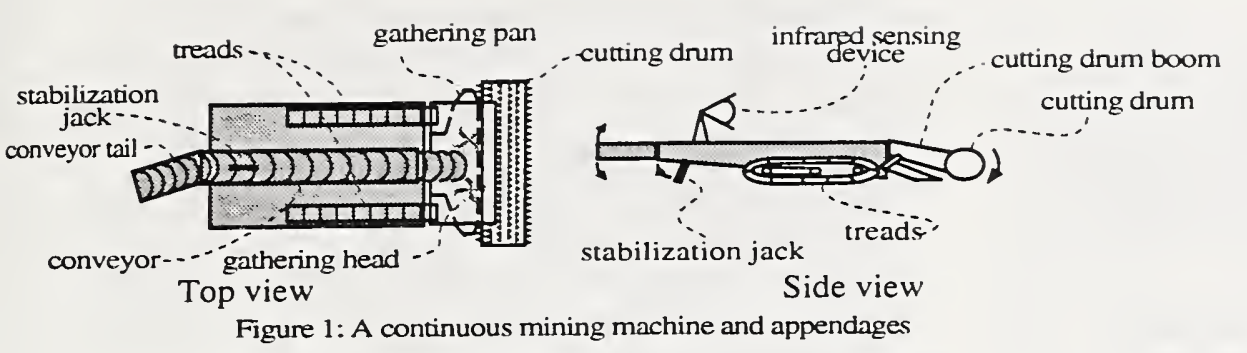

Figure 2: A continuous mining machine

two dimensions with certain minor adjustments for moving appendages (described in the section 3.1). Similarly, we can restrict the mine map to be two dimensional.

\subsection{REPRESENTATION METHODS FOR THE CONTINUOUS MINING MACHINE}

Continuous mining machines, as in figure 2, are common in underground coal mining. Bits on the rotating cutting drum cut into the coal seam while an on-board conveyance system moves the coal to the rear of the machine.

We are interested in the control and simulation of a specific continuous mining machine $(\mathrm{CM})$ called the JOY $14 \mathrm{CM}^{3}$. Figure 3 gives the relevant definitions and lengths required for motion simulation of this particular $\mathrm{CM}$ in an underground coal mine. As suggested in figure 3 , the $\mathrm{CM}$ is well represented as a polygonal hull, given our requirements. The movement of the conveyor tail and cutting drum boom (defined in figure 2) is discussed below.

We need to define finely spaced points along the polygonal hull representing the continuous mining machine in two dimensions. If $\mathbf{v}=\left(\mathbf{v}_{1}, \mathbf{v}_{2}, \ldots \mathbf{v}_{N}\right)$ is the $\mathrm{N}$ by 2 matrix of $\mathrm{CM}$ hull vertices,

$$
\mathbf{v}_{i}+k \Delta \frac{\mathbf{v}_{i+1}-\mathbf{v}_{i}}{\left\|\mathbf{v}_{i+1}-\mathbf{v}_{i}\right\|},
$$

can be used to define finely spaced points along the line segment between vertices $\mathbf{v}_{i}$

${ }^{3}$ The U.S. Bureau of Mines (USBM) is using JOY continuous mining machines as testbeds for computer controlled mining research. The JOY $14 C M$ is a product of JOY Manufacturing Corporation and reference to it does not constitute endorsement by NIST or the USBM.

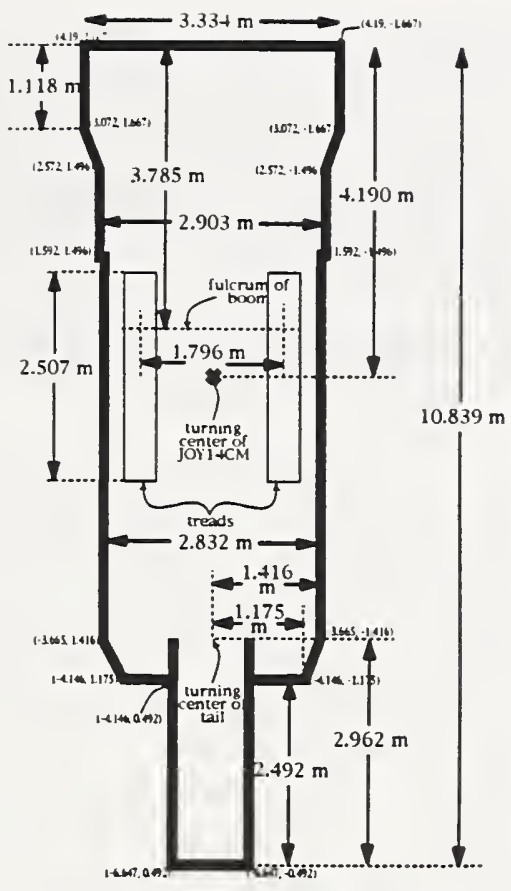

Figure 3: Important dimensions for determining the hull of a continuous mining machine

and $\mathbf{v}_{i+1}$ on the hull if we choose $\Delta$ such that $k \Delta<\left\|\mathbf{v}_{i+1}-\mathbf{v}_{i}\right\|$ for all $k=0,1, \ldots, k_{\max }$ [Hwang 90]. If $\Delta \geq\left\|\mathbf{v}_{i+1}-\mathbf{v}_{i}\right\|$, we just retain points $\mathbf{v}_{i}$ and $\mathbf{v}_{i+1}$. Each computing cycle, we use these points to test for 'collision' with an obstacle by indexing the occupancy grid with each point in (1) transformed into mine coordinates. Therefore, we only check grid locations along the $\mathrm{CM}$ hull. In our experiments, we checked occupancy probability values for approximately 100 points on the CM hull each computing cycle.

When the cutting drum boom is raised or lowered and/or the tail is moved (see figure 2), trigonometric computations are required to adjust the effected vertices. For example, the vertices where the conveyor tail meets the CM body are governed by (2) and (3). As illustrated in figure $2, \theta$ is the yaw of the tail (counterclockwise positive, measured off the negative $\mathrm{x}$-axis as defined in figure 3 ) in CM-centered coordinates. If $\theta$ is positive, $\mathbf{v}_{(N / 2)+1}$ doesn't change, and

$\mathbf{v}_{(N / 2)-2}(\theta)=$

$\mathbf{v}_{(N / 2)-2}(\theta=0)-(0,0.241 \tan \theta+0.492(\sec \theta-1))$.

If $\theta$ is negative, $\mathbf{v}_{(N / 2)-2}$ doesn't change, and 
$\mathbf{v}_{(N / 2)+1}(\theta)=$

$\mathbf{v}_{(N / 2)+1}(\theta=0)+(0,0.241 \tan |\theta|+0.492(\sec |\theta|-1))$.

Clearly, a 3D representation for the CM hull is required for high fidelity, but $2 \mathrm{D}$ is a sufficient first step.

\subsection{REPRESENTATION METHODS FOR THE MINE}

We originally chose to represent the mine in one way only, as a certainty grid [Moravec 88]. However, while seeking to simulate CM constrained motion, we quickly discovered that we needed to find the angle of incidence of the CM with the colliding wall at the collision point. This angle is not an easy quantity to obtain from a certainty grid. If we maintain a representation of the obstacle boundary in terms of approximate boundary points, this angle of incidence is easier to obtain. These approximate boundary points need to be oriented so that the occupied side of the boundary can be encoded by convention [Horst 94]. Furthermore, representing large sections of a mine in terms of a certainty grid is costly in terms of storage, whereas spatial occupancy representation in terms of obstacle boundary points requires significantly less storage. Therefore, we chose to maintain two representations of the mine map. One for local representation (the certainty grid) and one for global (the obstacle boundary map). A conversion algorithm is clearly needed and has been developed [Horst 94].

There are at least two types of mine maps in the system: 1) a simulated true map and 2) a best estimate map. This section will look at the former primarily, since that is all that was needed for this work.

The simulated true map is relatively easy to generate and use, since each cell in the grid representing the space is either occupied or not. Hence, the numerical values representing probability of occupancy are 0 or 1 within each cell. A best estimate map is that maintained in the world model at the servo level as in figure 1 (though map estimates will be also maintained at higher levels in the system). This map will register the uncertainty of occupancy at every cell through a probability of occupancy values between 0 and 1.
It is easier to maintain distances in a coordinate system chosen such that a unit square cell in the certainty grid is the unit of measurement. This reduces the amount of transformations needed and makes indexing the occupancy grid array simpler. For an occupancy grid near the mine 'face' (cutting area of a coal mine), each cell is chosen to be 0.1 meters square.

\section{CM MOTION SIMULATION}

The goal of this work is to simulate motion of a continuous mining machine (CM) in its environment (an underground coal mine) to the extent required. As we argue in the introduction, a simulation based on kinematics (i.e., translational and rotational velocities and accelerations without consideration of forces and torques) is preferred.

Here is the kinematics-based algorithm for simulated motion of the CM:

1) Move $\mathrm{CM}$ normally based on the command while checking for collision each computing cycle 4 . This means that each point on the CM hull is continually checked with the appropriate cell in the two dimensional certainty grid to see if it has occupancy probability higher than some threshold (equal to one in the simulated true map).

2) If a collision occurs, run a collision point filtering algorithm to determine whether the collision is one of four types:

a) one-point collision,

b) collision along a single 'cluster' of contiguous points

c) two-point collision, namely, collision at two or more non-contiguous locations.

d) collision along two separate clusters.

3) Using the object boundary representation, find the closest point on all boundary curves to the collision point,

4) Compute the angle of incidence of the $\mathrm{CM}$ with the obstacle boundary. This angle is defined by the $\mathrm{CM}$ orientation vector, the commanded motion, and the oriented obstacle boundary vector.

5) Based on the angle of incidence, the mine floor conditions, the commanded motion, and the type of collision,

a) if a one-point collision, determine whether the $\mathrm{CM}$ slides, rotates, or stops,

c) if a two-point collision, determine whether the CM slides or stops,

\footnotetext{
${ }^{4}$ Each software module has the standard RCS read, compute, write format for communications [Quintero 92].
} 
d) if a collision along cluster(s), determine whether the CM slides or stops.

\subsection{NORMAL MOTION}

Simulated normal motion is trivial without noise. If $\left(x_{k}, y_{k}, \psi_{k}\right)$ is the current position and orientation ${ }^{5}$ of the CM at time $k T$ and $T$ is the sampling period of the simulator, the updated position and orientation is,

$$
\begin{aligned}
& \left(x_{k+1}, y_{k+1}, \psi_{k+1}\right)= \\
& \left(x_{k}, y_{k}, \psi_{k}\right)+T\left(v_{k} \cos \psi_{k}, v_{k} \sin \psi_{k}, \omega_{k}\right),
\end{aligned}
$$

where $v_{k}$ is the translational velocity and $\omega_{k}$ is the rotational velocity at time $k T$. We expect that noise can be simulated easily with an additive random value with a non-zero bias. The bias could represented a slope in the mine floor. In the actual system, noise comes from a variety of sources, e.g., sensors, actuators, environment. We have not yet included noise in our simulation.

\subsection{SIMULATED COLLISION DETECTION AND ANGLE OF INCIDENCE COMPUTATION}

The collision detection portion within the kinematics-based CM motion simulator is now described. The current world coordinates of each point on the CM hull is continually checked against the corresponding cell in the two dimensional certainty grid to see if that cell is occupied. Our main complication here is that, even though there may only be a collision at one location, the simulator may detect that several points on the CM hull have collided. This happens if the value of the sampling period times velocity in (4) is large or if the chosen hull points (1) are closely spaced. Therefore, we developed a collision point filter that intelligently decides what type of collision occurred. The filter stores the number and location of each collision point and stores the number and locations of clusters of collision points when the CM has collided along an obstacle wall (a line segment in the obstacle boundary).

${ }^{5}$ Orientation is the angular difference between the $\mathrm{CM}$ reference frame and the global reference frame. The global reference frame is the $x-y$ coordinate axis.
Next the algorithm examines each collision point (or cluster) and determines which line segment in the obstacle boundary map represents the wall or walls against which the CM collided. This is done by computing the shortest distance to each of the line segments in the obstacle boundary map. The line segment corresponding to the smallest of these computed distances is chosen as the colliding wall.

The commanded value of motion to the $\mathrm{CM}$, the current orientation, and the vector of this closest line segment is then used to compute the angle of incidence. We define the angle of incidence as the smallest angle between the signed orientation (forward or reverse) and the vector of the closest line segment. If $u$ is the line segment vector and $v$ is the unit vector in the direction of the signed orientation of the CM, the angle of incidence, $\phi$, is

$$
\phi=\min \left\{\arccos \left(\frac{u \cdot v}{\sqrt{u \cdot u}}\right), \arccos \left(\frac{-u \cdot v}{\sqrt{u \cdot u}}\right)\right\} .
$$

$\phi$ is used to determine the type of motion (if any) following a collision.

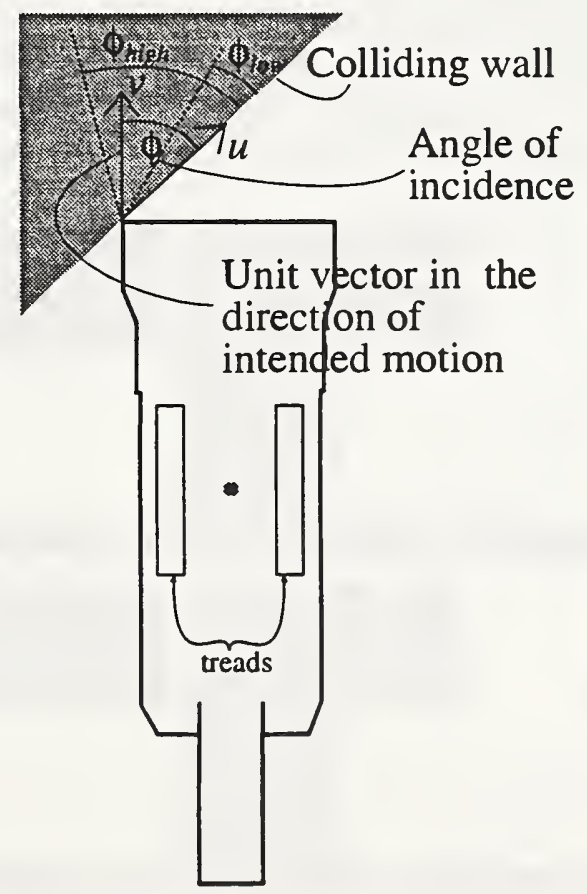

Figure 4: A continuous mining machine undergoing a single point collision 


\subsection{ONE POINT COLLISION}

The detection and consequence of a one point collision within the kinematics-based $\mathrm{CM}$ motion simulator is now described. The following algorithm is used. If the angle of incidence, $\phi$, is less than some lower threshold, say, $\phi_{\text {low }}$ (where $\phi_{\text {low }}$ is, at least, a function of the mine floor conditions and speed of the CM), we expect the CM to slide along the wall at the collision point. This motion involves a superposition of rotation about the collision point and translation along the colliding wall. If $\phi>\phi_{\text {high }}$, then we expect the CM to merely rotate about the collision point. Finally, if $\phi_{\text {low }} \leq \phi \leq \phi_{\text {high }}$ we expect no motion at all. This is particularly common in poor floor conditions (poor often means muddy). In such a situation the treads of the CM (see figure 2) can dig rather deeply into the mine floor. These definitions for CM motion after a single point collision are illustrated in figure 4.

\subsection{TWO POINT COLLISION}

The detection and consequence of a two point collision within the kinematics-based CM motion simulator is now described. The following algorithm is used. Find the locus of rotation by finding the intersection of the two lines perpendicular to the two colliding walls at the collision points. Rotate the CM about this locus. Translate towards each wall enough to compensate for each rotation. Such motion is suggested in figure 5 .

\subsection{OTHER TYPES OF COLLISION}

The effect of other types of collisions within the kinematics-based CM motion simulator is now described. If there is a collision with a single cluster of points and the vector formed by the cluster is parallel to the intended motion, slide the CM along the wall. If there is a collision with a single cluster of points and the vector formed by the cluster is perpendicular to the intended motion, stop the CM. If more than one cluster is detected, stop.

\section{CONCLUSION}

We report on a simulator for the motion of a particular continuous mining machine $(\mathrm{CM})$ in a coal mine. We particularly focus on the motion of this $\mathrm{CM}$ following a collision with an immovable obstacle (typically a coal rib or face). The unique requirements of $\mathrm{CM}$ motion simulation effects our choice of the representations of the $\mathrm{CM}$ and the mine environment in the following ways:

1) We chose to represent the $\mathrm{CM}$ as a two dimensional polygonal hull.

2) The mine obstacles are given dual representations, each of which are advantageous in the efficient performance of specific tasks. An object boundary curve representation is most efficient in determining motion following a collision and the certainty grid is more efficient for determining whether and where a collision has occurred.
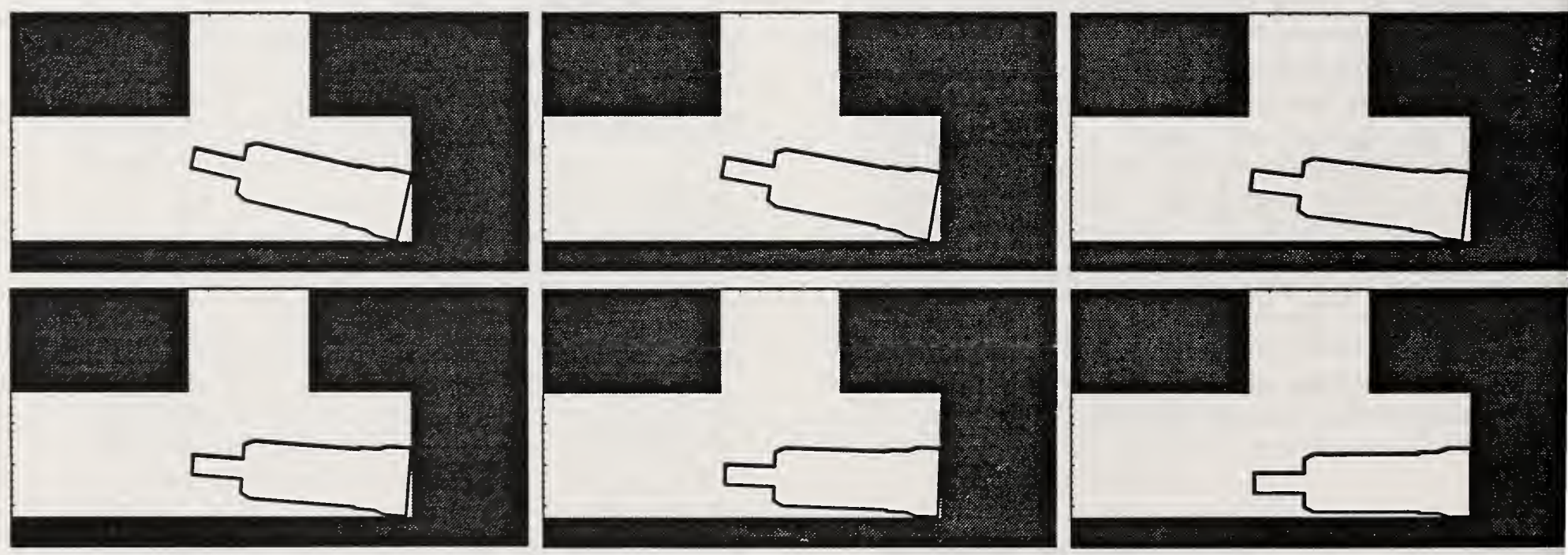

Figure 5: Motion following a two point collision 
We model normal motion of the CM without noise, but specify how it would be included, namely, the effect of the mine floor and sensor noise on CM position and orientation can be simulated by a non-zero mean random number added to the simulated 'true' value. If a collision is detected, abnormal motion ensues. The type of collision, the mine floor conditions, and the commanded motion are among those factors influencing the subsequent motion of the CM.

We could extend this work into three dimensions, but the amount of extra effort was beyond our resources and requirements. An intermediate step might be to consider 'two and a half' dimensions, i.e., where the heights of things are added to the two dimensional representation. Finally, we didn't handle reverse, rotate, or pivot type motions of the CM.

\section{REFERENCES}

[Horst 93] Horst, J. A., “An intelligent control system for a cutting operation of a continuous mining machine", NISTIR, National Institute of Standards and Technology, U.S. Department of Commerce, 1993.

[Horst 94] Horst, J. A., "A certainty grid to object boundary algorithm", NIST Interagency report in progress.

[Hwang 90] Hwang, Yong K., "Boundary equations of configuration obstacles for manipulators",

[Moravec 88] Moravec, Hans P., "Sensor fusion in certainty grids for mobile robots", AI Magazine, Summer 1988, 61-74.

[Schiffbauer 92] Schiffbauer, W. H., "Flexible control networks in mining machines to improve worker safety", IEEE Control Systems Magazine, June 1992. 

